\title{
SEGUIMENTO DO ENFERMEIRO GRADUADO NA ESCOLA DE ENFERMAGEM DA USP: SUA INSERÇÃO EM UNIDADES DE TERAPIA INTENSIVA
}

\section{FOLLOWING UP OF THE NURSES GRADUATED AT THE SCHOOL OF NURSING OF THE UNIVERSITY OF SÃO PAULO: THEIR INSERTION IN INTENSIVE CARE UNITS}

\author{
Paula Hatsuye Kitahara* \\ Miako Kimura** \\ Katia Grillo Padilha**
}

KITAHARA, P.H.; KIMURA, M.; PADILHA, K.G. Seguimento do enfermeiro graduado na Escola de Enfermagem da USP: sua inserção em Unidades de Terapia Intensiva. Rev.Esc.Enf.USP, v.33, n.3, p.284-93, set. 1999.

\begin{abstract}
RESUMO
O estudo foi realizado com os objetivos de identificar as atividades desenvolvidas após a formatura pelos enfermeiros graduados na Escola de Enfermagem da USP (EEUSP) e caracterizar a inserção destes enfermeiros em Unidades de Terapia Intensiva (UTIs) no que se refere a ter sido ou não sua primeira área de atuação, à experiência anterior de trabalho em UTI, ao treinamento inicial específico e às dificuldades enfrentadas para assumir as atividades nessas Unidades. A população foi composta pelos egressos da EEUSP nos anos de 1991 a 1995 . Uma listagem dos alunos formados no período, com os respectivos endereços e telefones, foi obtida junto à Seção de Graduação. Foi utilizado um questionário para a coleta de dados. Dentre os 235 questionários enviados, 117 (49,8\%) retornaram com resposta. Quanto as atividades após a formatura, 90,5\% informaram estar exercendo a profissáo; o hospital foi o local de trabalho mais indicado, totalizando $76,2 \%$ das respostas. Em todos os anos do periodo estudado, um contingente significativo de enfermeiros foi absorvido nas UTIs. Dos 117 respondentes, 55 (47,0\%) informaram que trabalham ou trabalharam em UTI, sendo que para $54,5 \%$ deles, este foi o primeiro emprego. A maioria (52,8\%) não tinha experiência de trabalho anterior em UTI. Apesar de 64,1\% dos enfermeiros terem recebido treinamento inicial específico, foram mencionadas dificuldades para assumir as atividades na UTI, dentre as quais destacaram-se: a falta de destreza manual e de conhecimento teórico $(45,4 \%)$, o manuseio de equipamentos $(25,8 \%)$ e o relacionamento com as equipes de enfermagem e multiprofissional $(8,2 \%)$.
\end{abstract}

UNITERMOS: Educação em enfermagem. Exercício da enfermagem. Unidades de terapia intensiva.

\begin{abstract}
The purposes of this research was to identify the activities developed by the nurses after undergraduation course at the School of Nursing of the University of São Paulo and to characterize their insertion in Intensive Care Units (ICUs), in relation to have been or not their first area of job, their previous experience in ICU, the specific initial training and difficulties faced to take on the ICU activities. The population was composed by nurses graduated between $199 \mathrm{l}$ and 1995. A rol of names, addresses and telephone numbers was obtained at the school office. Two hundred and thirty five questionnaires were sent by mail and $117(49,8 \%)$ were returned. The majority of the respondents $(90,5 \%)$ were working as nurses, mainly in hospitals, which was the most indicated area of work (76,2\%). Almost half of the informants (47,0\%) said they worked or were working in ICUs and for 54,5\% of them, this was their first job; $52,8 \%$ had no previous experience in ICU. Although $64,1 \%$ of these nurses had received a specific initial training, some difficulties to take on the ICU activities were faced: lack of knowledge and psychomotor skills $(45,4 \%)$, handling equipments $(25,8 \%)$ and relationship with the multiprofessional and the nursing team (8,2\%).
\end{abstract}

UNITERMS: Education, Nursing. Nursing profession. Intensive care units.

* Aluna do Curso de Graduação da Escola de Enfermagem da Universidade de São Paulo.Bolsista PIBIC-CNPq/USP.

** Professor Livre Docente do Departamento de Enfermagem Médico-Cirúrgica da Escola de Enfermagem da USP 


\section{INTRODUÇÃO}

A inserção do graduado em enfermagem no campo de trabalho tem sido motivo de preocupação dos profissionais ligados à docência e aos serviços. Tal preocupação traduz o compromisso comum de todos que atuam nas instâncias responsáveis pela formação e pelo desempenho no exercício profissional do graduado em enfermagem.

Ao término do Curso de Graduação, o enfermeiro recém-formado enfrenta o impacto da realidade profissional que, com freqüência, é geradora de ansiedade. Inúmeras dificuldades estão presentes neste início de vida profissional, principalmente aquelas relacionadas à falta de correlação entre teoria e prática, à falta de habilidade para a execução das tarefas e para o desempenho de funções preestabelecidas pela instituição hospitalar (IDE et al, 1985; KREUTZ, 1996).

Nos últimos anos temos observado, com certa freqüência, que enfermeiros recém-formados estão sendo chamados para a prática em Unidades de Terapia Intensiva (UTIs), a despeito de controvérsias sobre a adequação desta área para enfermeiros iniciantes.

Segundo OERMANN (1991) e KURCGANT (1991) a complexidade do paciente de U'TI requer do enfermeiro uma base sólida de conhecimentos, habilidade nos aspectos tecnológicos e emocionais do cuidado, habilidade para fazer julgamentos clínicos frente a situações que se alteram rapidamente, além de um sistema de valores que o leve a tomar decisões éticas na prática diária. $O$ desenvolvimen to destes requisitos e competências é um processo gradual e, tradicionalmente, só profissionais com maior experiência têm sido selecionados para a assistência ao paciente crítico.

Em experiências passadas, o ensino de enfermagem em UTI era iniciado já na Graduação, como uma sub-unidade da disciplina Enfermagem Médico-Cirúrgica, no 4o. semestre do Curso, embora não constasse formalmente da grade curricular (KIMURA et al., 1986). Apesar de terem sido estágios bastante valorizados pelos alunos, este conteúdo deixou de ser ministrado a partir de 1986 , em decorrência de dificuldades operacionais, tanto dos campos de prática como do grupo de docentes. Com a implementação do novo currículo de graduação na Escola de Enfermagem da USP (EEUSP), o ensino de enfermagem em UTI passa a integrar a grade curricular, como disciplina oferecida no $7^{\circ}$ semestre.

Aspectos importantes como as especificidades regionais e a legislação sobre o exercício profissional da enfermagem justificam a inclusão desse conteúdo na formação do enfermeiro. Estudos realizados por IDE; CHAVES (1989) e por KIMURA; MIYADAHIRA (1991) constataram uma acentuada concentração de UTIs na Região Sudeste, no Estado e no Município de São Paulo o que, por si só, impõe às escolas a necessidade do ensino da assistência de enfermagem ao doente crítico internado em UTI. As características sócio-econômicas, demográficas e do perfil epidemiologico das citadas regiōes favoreceram um maior desenvolvimento da assistência intensiva, configurando uma demanda assistencial e um mercado de trabalho bem definidos para o enfermeiro, que necessita de base técnico-científica solida para o desempenho profissional junto aos pacientes graves.

Além disso, a capacitação dos enfermeiros para atuar em UTI também faz-se imprescindível se considerarmos que a Lei 7498/86, em seu artigo 11, determina como sendo de competência privativa do enfermeiro " prestar cuidados diretos de enfermagem a pacientes graves, em risco de vida" (BRASIL, 1986). Dessa forma, o aspecto legal do exercício profissional rafirma também a necessidade de conteúdos específicos sobre UTI no ensino de enfermagem.

No momento em que se vivencia um marco importante do ensino de graduação em enfermagem, sobretudo no âmbito do ensino da assistência em UTI, acreditamos ser oportuna a proposição deste estudo com a finalidade de analisar como tem sido, nos últimos anos, a inserção do enfermeiro graduado nesta Escola, no campo de trabalho, mais especificamente em UTI.

São, portanto, objetivos deste estudo:

-Identificar as atividades desenvolvidas pelo enfermeiro graduado na EEUSP, após a formatura.

- Caracterizar a inserção do enfermeiro graduado nesta Escola em UTI, no que se refere a: ter sido ou não a sua primeira área de atuação, experiência anterior de trabalho na UTI, realização ou não de treinamento inicial específico e dificuldades enfrentadas para assumir as funções como enfermeiro de UTI.

\section{METODOLOGIA}

\subsection{População}

A população deste trabalho foi composta pelos enfermeiros formados na EEUSP no período de 1991 a 1995. A listagem, por ano, do total de 256 enfermeiros formados no período, com os respectivos endereços e telefones foi obtida junto à Seção de Graduação da Escola. 


\subsection{Coleta de Dados}

Instrumento: Foi utilizado um questionário (Anexo I) com questões abertas e fechadas, contendo duas partes descritas a seguir:

Parte I: Dados gerais - destinada a obter informações sobre idade, sexo, experiência de trabalho na enfermagem durante a graduação e cursos realizados após a formatura.

Parte II: Dados específicos - nesta parte, foram obtidos dados relativos às atividades desenvolvidas na enfermagem, após a formatura, particularmente as relacionadas à inserção e atuação em UTIs.

Procedimentos: Mediante contato prévio por telefone, foram confirmados os endereços dos enfermeiros, para os quais foram enviados os questionários acompanhados de uma carta com informações sobre o estudo.
Foi também enviado um envelope selado para a devolução do questionário, estabelecendo-se o prazo de um mês contado a partir da data de postagem.

\subsection{Tratamento de Dados}

Os dados obtidos foram tabulados e distribuídos em tabelas e analisados com base em números absolutos e percentuais. As respostas abertas foram agrupadas de acordo com suas semelhanças.

\section{RESULTADOS E DISCUSSÃO}

A Tabela 1 apresenta a distribuição, por ano, do número de enfermeiros formados no período, dos questionários enviados e respectiva proporção de respostas recebidas.

Tabela 1. Enfermeiros formados na EEUSP entre 1991 e 1995, segundo o número de questionários enviados e respondidos e o ano de formatura. São Paulo, 1996.

\begin{tabular}{ccccc}
\hline Ano & $\begin{array}{c}\text { Enfermeiros } \\
\text { Formados }\end{array}$ & $\begin{array}{c}\text { Questionários } \\
\text { Enviados }\end{array}$ & \multicolumn{2}{c}{$\begin{array}{c}\text { Questionários } \\
\text { respondidos }\end{array}$} \\
\hline 1991 & 27 & 26 & 15 & $(57,7 \%)$ \\
1992 & 51 & 44 & 27 & $(61,4 \%)$ \\
1993 & 56 & 47 & 29 & $(61,7 \%)$ \\
1994 & 61 & 57 & 21 & $(36,8 \%)$ \\
1995 & 61 & 61 & 25 & $(41,0 \%)$ \\
\hline Total & 256 & 235 & 117 & $(49,8 \%)$ \\
\hline
\end{tabular}

Do total de 256 enfermeiros formados no período, 21 foram excluídos previamente pelos seguintes motivos: 11 por mudança de telefone/ endereço; 9 por estarem fora do país e 1 por ter-se recusado a participar. Foram, assim, enviados 235 questionários. Destes, 8 foram devolvidos ao remetente sem resposta por não terem sido localizados os destinatários e $117(49,8 \%)$ retornaram respondidos; foram estes, portanto, os questionários considerados no presente estudo. Observa-se que os percentuais de retorno foram maiores entre os alunos dos três primeiros anos investigados (91,92 e 93), quando comparados aos dos anos mais recentes (94 e 95).

Quanto à caracterização da faixa etária dos enfermeiros respondentes, observou-se que 54,3\% tinham idade entre 20 e 23 anos à época da formatura. Considerando que o curso de graduação em enfermagem tem duração de quatro anos, podese inferir que a maioria dos enfermeiros deste estudo ingressou na Universidade com idade entre 17 e 20 anos, época que, em geral, alunos com trajetória escolar normal, realizam o exame vestibular.

No estudo de NAKAMAE (1976), que comparou o perfil dos estudantes de enfermagem do Estado de São Paulo nos anos de 1973 e 1976, já se constatava a tendência de um número cada vez maior de estudantes com escolaridade normal.

Corroborando a hegemonia do sexo feminino na enfermagem, os enfermeiros do presente estudo apresentaram também um perfil quase que exclusivamente feminino $(98,3 \%)$.

Nas Tabelas 2, 3 e 4 apresentadas a seguir, podem-se observar as características dos enfermeiros formados no período considerado, quanto a sua experiência de trabalho durante a graduação, a continuidade dos estudos após a formatura e aos locais de atuação profissional. 
Tabela 2. Experiência de trabalho na enfermagem durante a graduação, segundo o ano de formatura. São Paulo, 1996.

\begin{tabular}{|c|c|c|c|c|c|c|}
\hline \multirow{3}{*}{ Ano } & \multicolumn{4}{|c|}{ Experiência de trabalho durante a graduação } & \multirow{2}{*}{\multicolumn{2}{|c|}{ Total }} \\
\hline & \multicolumn{2}{|c|}{ Sim } & \multicolumn{2}{|c|}{ Não } & & \\
\hline & $\mathrm{n}$ & $\%$ & $\mathrm{n}$ & $\%$ & $\mathrm{n}$ & $\%$ \\
\hline 1991 & 10 & 66,7 & 5 & 33,3 & 15 & 100,0 \\
\hline $1992^{*}$ & 18 & 69,2 & 8 & 30,8 & 26 & 100,0 \\
\hline 1993 & 25 & 86,2 & 4 & 13,8 & 29 & 100,0 \\
\hline 1994 & 17 & 81 & 4 & 19 & 21 & 100,0 \\
\hline 1995 & 12 & 48 & 13 & 52 & 25 & 100,0 \\
\hline Total & 82 & 70,7 & 34 & 29,3 & 116 & 100,0 \\
\hline
\end{tabular}

*Um questionário sem informaçăo

Verifica-se que nos analisados, a maioria dos enfermeiros respondentes $(70,7 \%)$ teve alguma experiência de trabalho na enfermagem enquanto alunos de graduação. Com exceção do grupo de 1995 , em que $52,0 \%$ informou não ter tido outra atividade concomitante à graduação, nos demais anos houve sempre predominância dos que trabalharam durante o curso, com destaque para o ano de $1993(86,2 \%)$.

Dentre as atividades extracurriculares desenvolvidas pelos 82 enfermeiros com experiência de trabalho na enfermagem durante a faculdade, o estágio remunerado, aqui considerado como uma atividade laborativa, foi mencionado de maneira preponderante sobre as demais atividades, totalizando $67,7 \%$ das respostas. O estágio voluntário $(13,5 \%)$ e o trabalho como técnico de enfermagem $(12,5 \%)$ foram as atividades indicadas a seguir.

Embora esses dados possam refletir, de um lado, as dificuldades econômicas dos alunos (necessidade de trabalhar), por outro lado, parecem indicar uma preocupação em buscar outras experiências além daquelas proporcionadas pelo ensino formal, que lhes possibilitassem melhor preparo e maior segurança na prestação da assistência aos pacientes.

Tabela 3. Cursos realizados após a formatura pelos enfermeiros, segundo o ano de formatura. São Paulo, 1996.

\begin{tabular}{|c|c|c|c|c|c|c|c|}
\hline \multirow{3}{*}{ Cursos } & \multicolumn{5}{|c|}{ Ano de formatura } & \multirow{2}{*}{\multicolumn{2}{|c|}{ Total }} \\
\hline & \multirow[t]{2}{*}{91} & \multirow[t]{2}{*}{92} & \multirow[t]{2}{*}{93} & \multirow[t]{2}{*}{94} & \multirow[t]{2}{*}{95} & & \\
\hline & & & & & & $\mathrm{n}$ & $\%$ \\
\hline Especialização & 14 & 15 & 4 & 5 & 3 & 41 & 41,0 \\
\hline Atualização & 6 & 6 & 9 & 6 & 7 & 34 & 34,0 \\
\hline Licenciatura & 1 & 4 & 2 & 5 & 2 & 14 & 14,0 \\
\hline Aprimoramento & 1 & - & 1 & 3 & 2 & 7 & 7,0 \\
\hline Mestrado & - & $\cdot$ & 2 & 2 & - & 4 & 4,0 \\
\hline Total & 22 & 25 & 18 & 21 & 14 & 100 & 100,0 \\
\hline
\end{tabular}

Os cursos especificados na Tabela 3 referemse às respostas de 81 enfermeiros $(69,2 \%)$ que realizaram algum tipo de curso após a graduação. Chama à atenção o fato de que $30,8 \%$ deles informou não ter feito nenhum curso após a formatura. Conforme se pode observar, dos 100 cursos mencionados, os de Especialização foram os mais indicados pelos enfermeiros deste estudo $(41,0 \%)$, seguidos dos cursos de Atualização $(34,0 \%)$ e de Licenciatura (14,0\%). Os cursos menos freqüentados foram os de Mestrado (4,0\%).

Dentre os cursos de Especialização, os mais freqüentemente citados foram os de Enfermagem em UTI (9), de Enfermagem do Trabalho (9), de 
Administração Hospitalar (7) e de Enfermagem Obstétrica (4).

Quanto as atividades desenvolvidas após a formatura, dos 116 enfermeiros que responderam a esta questão, $105(90,5 \%)$ informaram que exerciam a profissão, o que reflete um baixo percentual de evasão profissional. Quanto aos demais enfermeiros, $5(4,3 \%)$ haviam abandonado a enfermagem, $3(2,6 \%)$ nunca trabalharam como enfermeiros e $3(2,6 \%)$ apenas continuavam a estudar ou eram bolsistas em projetos de pesquisa de enfermagem.

Tabela 4. Locais de atuação profissional dos enfermeiros após a formatura, segundo o ano de formatura. São Paulo, 1996.

\begin{tabular}{|c|c|c|c|c|c|c|c|c|c|c|c|c|}
\hline \multirow{3}{*}{ Locais } & \multicolumn{10}{|c|}{ Ano de formatura } & & \\
\hline & \multicolumn{2}{|c|}{91} & \multicolumn{2}{|c|}{92} & \multicolumn{2}{|c|}{93} & \multicolumn{2}{|c|}{94} & \multicolumn{2}{|c|}{95} & \multicolumn{2}{|c|}{ Total } \\
\hline & $\mathrm{n}$ & $\%$ & $\mathrm{n}$ & $\%$ & $\mathrm{n}$ & $\%$ & $\mathrm{n}$ & $\%$ & $\mathrm{n}$ & $\%$ & $\mathrm{n}$ & $\%$ \\
\hline Hospital & 15 & 78,9 & 27 & 69,2 & 27 & 73,0 & 21 & 77,8 & 22 & 88,0 & 112 & 76,2 \\
\hline Escola & 2 & 10,5 & 9 & 23,1 & 5 & 13,5 & - & - & 2 & 8,0 & 18 & 12,2 \\
\hline Indústria & 1 & 5,3 & 2 & 5,1 & 2 & 5,4 & 1 & 3,7 & $\cdot$ & - & 6 & 4,1 \\
\hline Clínica particular & 1 & 5,3 & 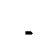 & - & 2 & 5,4 & 1 & 3,7 & $\cdot$ & - & 4 & 2,7 \\
\hline Outros* & $\cdot$ & - & 1 & 2,6 & 1 & 2,7 & 4 & 14,8 & 1 & 4 & 7 & 4,8 \\
\hline Total & 19 & 100,0 & 39 & 100,0 & 37 & 100,0 & 27 & 100,0 & 25 & 100,0 & 147 & 100,0 \\
\hline
\end{tabular}

* Outros: Fundação Instituto de Pesquisas Econômicas (1-94; 1-95); Unidade Básica de Saúde (1-94); Secretaria da Saúde (1-93) Curso ATLS para médicos (1.94); Atendimento em condomínio de férias (1-92); Banco de Sangue (1-94).

Verifica-se na Tabela 4, que o hospital foi a área de trabalho mais citada como local de atuação pelos enfermeiros formados na EEUSP em todos os anos analisados neste estudo, totalizando $76,2 \%$ das indicações. Observa-se, também, que $12,2 \%$ dos profissionais atuaram na área de ensino de enfermagem, sobretudo em cursos para auxiliares de enfermagem (12 das 18 indicações). Além desses, foram também mencionadas atuação na Indústria $(4,1 \%)$, em Clínicas particulares $(2,7 \%)$, bem como em áreas diversas, o que denota o amplo leque de possibilidades de atuação dos enfermeiros no mercado de trabalho, embora ainda com pequena frequência.

Nas Tabelas a seguir, estão apresentados os resultados referentes à inserção dos enfermeiros em Unidades de Terapia Intensiva.

Tabela 5. Enfermeiros que atuam ou atuaram em UTI, segundo o ano de formatura. São Paulo, 1966.

\begin{tabular}{c|ccc}
\hline & Enfermeiros & \multicolumn{2}{c}{$\begin{array}{c}\text { Enfermeiros que atuam } \\
\text { Respondentes }\end{array}$} \\
\hline 1991 & 15 & 9 & $(60,0 \%)$ \\
1992 & 27 & 14 & $(51,9 \%)$ \\
1993 & 29 & 10 & $(34,5 \%)$ \\
1994 & 21 & 9 & $(42,9 \%)$ \\
1995 & 25 & $13 \quad(52,0 \%)$ \\
\hline Total & 117 & $55 \quad(47,0 \%)$ \\
\hline
\end{tabular}

Verifica-se que, dos 117 enfermeiros respondentes, $55(47,0 \%)$ atuam ou atuaram em UTI após a formatura. É interessante observar que, em todos os anos do período estudado, um contingente significativo de enfermeiros foi absorvido nas UTIs com percentuais acima de $50,0 \%$ nos anos de 91 $(60,0 \%), 92(51,9 \%)$, e $95(52,0 \%)$. 
Tabela 6. Distribuição dos enfermeiros que atuam ou atuaram em UTI como $1^{\circ}$ emprego e ano de formatura. São Paulo, 1996.

\begin{tabular}{|c|c|c|c|c|c|c|}
\hline \multirow{3}{*}{ Ano } & \multicolumn{4}{|c|}{ UTI como $1^{\circ}$ emprego } & \multirow{2}{*}{\multicolumn{2}{|c|}{ Total }} \\
\hline & \multicolumn{2}{|c|}{$\operatorname{Sim}$} & \multicolumn{2}{|c|}{ Não } & & \\
\hline & $\mathrm{n}$ & $\%$ & $\mathrm{n}$ & $\%$ & $\mathrm{n}$ & $\%$ \\
\hline 1991 & 5 & 55,6 & 4 & 44,4 & 9 & 100,0 \\
\hline 1992 & 6 & 42,9 & 8 & 57,1 & 14 & 100,0 \\
\hline 1993 & 5 & 50,0 & 5 & 50,0 & 10 & 100,0 \\
\hline 1994 & 5 & 55,6 & 4 & 44,4 & 9 & 100,0 \\
\hline 1995 & 9 & 69,2 & 4 & 30,8 & 13 & 100,0 \\
\hline Total & 30 & 54,5 & 25 & 45,5 & 55 & 100,0 \\
\hline
\end{tabular}

Observa-se, na Tabela 6 , que para $54,5 \%$ dos enfermeiros que atuam ou atuaram na UTI após a formatura, esta foi a primeira área de atuação. Esta predominância foi observada sobretudo no ano de 1995, quando aproximadamente $70 \%$ dos formados atuaram na UTI, como seu primeiro emprego.

É importante ressaltar que mais da metade dos 55 enfermeiros $(52,8 \%)$ informou que não tinha qualquer tipo de experiência anterior de trabalho em UTI. Os demais enfermeiros $(47,2 \%)$ relataram que a experiência anterior com a assistência intensiva foi adquirida em estágios remunerados (54,2\%), como técnico de enfermagem $(20,8 \%)$, em estágios voluntários $(12,5 \%)$, como auxiliares de enfermagem $(8,3 \%)$ e em estágio curricular de administração em enfermagem $(4,2 \%)$.

Diversos autores têm analisado a questão da admissão de enfermeiros recém-formados nas UTIs (CANFIELD, 1982; GOTTSCHALL et al, 1983; OERMANN, 1991; BURNS; HUTCHENS, 1992). Embora existam controvérsias a esse respeito, verificou-se que muitos dos hospitais incluídos nos estudos citados empregavam enfermeiros sem experiência em cuidados intensivos, inclusive, recémformados. Nestes estudos, o percentual de hospitais que adotavam tal prática variou de $40 \%$ a $87 \%$.

Tabela 7. Distribuição dos enfermeiros segundo treinamento inicial específico para atu:ar na UTI e ano de formatura. São Paulo, 1996.

\begin{tabular}{|c|c|c|c|c|c|c|}
\hline \multirow{3}{*}{ Ano } & \multicolumn{4}{|c|}{ Treinamento inicial específico } & \multirow{2}{*}{\multicolumn{2}{|c|}{ Total }} \\
\hline & \multicolumn{2}{|c|}{ Sim } & \multicolumn{2}{|c|}{ Não } & & \\
\hline & $\mathrm{n}$ & $\%$ & $\mathrm{n}$ & $\%$ & $\mathrm{n}$ & $\%$ \\
\hline 1991 & 5 & 55,6 & 4 & 44,4 & 9 & 100,0 \\
\hline $1992^{*}$ & 8 & 61,5 & 5 & 38,5 & 13 & 100,0 \\
\hline 1993 & 6 & 60,0 & 4 & 40 & 10 & 100,0 \\
\hline 1994 & 6 & 66,7 & 3 & 33,3 & 9 & 100,0 \\
\hline $1995^{*}$ & 9 & 75,0 & 3 & 25,0 & 12 & 100,0 \\
\hline Total & 34 & 64,2 & 19 & 35,8 & 53 & 100,0 \\
\hline
\end{tabular}

*Um questionário sem informação

Observa-se na Tabela 7 que, dentre os enfermeiros com atuação em UTI, a maioria (64,2\%) recebeu treinamento inicial específico para o trabalho nessa Unidade.

Nota-se, também, em todos os anos analisados, elevado percentuail de enfermeiros que receberam esse tipo de treinamento ocorrendo, a partir de 93, um aumento progressivo desse percentual.

O treinamento dos enfermeiros recém. admitidos em unidades específicas faz parte de um programa de orientação mais abrangente oferecido pelas instituições, visando a introdução e a 
adaptação do novo funcionário em seu local de trabalho e auxiliando-o na aquisição de habilidades e conhecimentos especificos dessa Unidade.

WIRCHOWISKI; KUBSCH (1995) alertam sobre a importância de se proporcionar programas educacionais que atendam adequadamente às necessidades do enfermeiro de UTI, dada a complexidade da assistência ao paciente crítico e a presença de alta tecnologia.

Em levantamento realizado nos hospitais com UTI dos EUA, SULLIVAN; BREU (1982) verificaram que $83 \%$ deles ofereciam programas de orientação em serviço para a equipe de enfermagem.

No estudo de KOIZUMI et al (1997), abrangendo 43 U'TIs do Município de São Paulo, constatou-se que em 79,1\% delas havia um programa específico para o início das atividades do enfermeiro na Unidade. A comparação desse percentual com o de 46,4\% obtido em estudo anterior (GOMES et al, 1987), evidencia uma crescente preocupação das instituições com o preparo inicial dos enfermeiros de UTI, embora ainda se tenha observado, no presente estudo, que $35,8 \%$ dos enfermeiros assumiram suas funções sem treinamento prévio. Tal fato certamente traz dificuldades ao desempenho profissional do enfermeiro, podendo comprometer a qualidade da assistência prestada.

Para a maioria dos respondentes deste estudo, a duração do treinamento foi de 30 dias. O período mais freqüentemente encontrado nas UTIs estudadas por KOIZUMI et al (1997) foi igualmente o de até um mês, não se tendo observado modificações nos últimos anos quanto a esse aspecto, comparativamente aos resultados de GOMES et al (1987).

Outros estudos serão necessários para se averiguar se esse período tem sido suficiente para o treinamento inicial na Unidade. É preciso considerar, entretanto, que tal treinamento é apenas uma etapa inicial do processo de formação do enfermeiro intensivista, processo esse que deve ser assumido tanto como um compromisso individual de cada profissional, como uma responsabilidade institucional.

Tabela 8. Conteúdo abordado durante o treinamento específico dos enfermeiros para atuação na UTI. São Paulo, 1996.

\begin{tabular}{lcc}
\hline Conteúdo & $\mathrm{n}$ & $\%$ \\
\hline Aspectos técnicos da assistência & 29 & 43,2 \\
Aspectos administrativos & 25 & 37,3 \\
Avaliação das condiçres físicas do paciente & 4 & 6,0 \\
Revisão de fisiopatologia & 3 & 4,5 \\
Medicaçס̃es & 3 & 4,5 \\
Aspectos psicossociais da assistência ao paciente e à família & 2 & 3,0 \\
Postura profissional & 1 & 1,5 \\
\hline Total
\end{tabular}

Os dados desta Tabela evidenciam uma ênfase em conteúdos voltados para os aspectos técnicos $(43,2 \%)$ e administrativos da UTI $(37,3 \%)$. Entre os primeiros, foram destacados o uso de equipamentos e materiais, como respiradores, monitores e catéteres e a execução de procedimentos técnicos no cuidado direto ao paciente. Nos aspectos administrativos, foram mencionadas as orientações gerais sobre a planta física e a dinâmica da UTI, a confecção de relatórios, estatísticas e as rotinas da Unidade. Aspectos igualmente relevantes para a assistência ao paciente crítico, como a avaliação de suas condições físicas e a fundamentação dos processos fisiopatologicos, bem como os aspectos psicossociais da assistência ao paciente e à família foram pouco abordados. Resultados semelhantes foram encontrados no estudo de KOIZUMI et al (1997) e CHABOYER et al (1997).

Tabela 9. Dificuldades enfrentadas para assumir as funções como enfermeiro de UTI. São Paulo, 1996.

\begin{tabular}{lcc}
\hline Dificuldades & $\mathrm{n}$ & $\%$ \\
\hline Falta de destreza manual e de conhecimento teórico & 44 & 45,4 \\
Desconhecimento do manuseio de equipamentos & 25 & 25,8 \\
Relacionamento com equipes de enfermagem e multiprofissional & 8 & 8,2 \\
Avaliação do paciente & 5 & 5,1 \\
Desconhecimento de rotinas/dinâmica de UTI & 3 & 3,1 \\
Número elevado de pacientes & 3 & 3,1 \\
Falta de estímulo para o desenvolvimento da enfermagem & 3 & 3,1 \\
Falta de visão da UTI como um todo & 2 & 2,1 \\
Outras* & 4 & 4,1 \\
\hline Total & 97 & 100,0 \\
\hline
\end{tabular}

* Outras: Falta de pessoal (1); Implantação de normas e rotines (1); Compatibilização das funçoes administrativas e assistencias 
Conforme se observa na Tabela 9, a principal dificuldade enfrentada pelos enfermeiros ao assumirem as funções na UTI foi a falta de destreza manual e de conhecimento teorico para prestar assistência de enfermagem a pacientes graves $(45,4 \%)$. Os enfermeiros mencionaram a falta desse preparo sobretudo quanto à atuação em situações de emergência e de outras intercorrências e à diversidade de procedimentos e medicações requeridos pelo paciente grave. O manuseio de equipamentos representou $25,8 \%$ do total de dificuldades mencionadas

É interessante notar que as dificuldades relacionadas às habilidades e conhecimentos técnicos foram preponderantes a despeito de terem sido justamente estes os aspectos mais abordados durante a fase de treinamento. Pode-se, assim, supor que o período de um mês a ele destinado tenha sido insuficiente para o domínio de toda a gama de habilidades e conhecimentos que habitualmente se espera do enfermeiro de UTI. Isso se torna particularmente relevante se lembrarmos que mais da metade dos profissionais que se destinaram às UTIs, não tinham experiência anterior nessa área e que nenhum deles teve esse conteúdo no ensino formal, durante a graduação.

\section{CONCLUSÕES}

Os resultados do presente estudo permitem concluir que:

- os 117 enfermeiros participantes eram predominantemente do sexo feminino $(98,3 \%)$, com idlade entre 20 e 23 anos a época de formatura $(54,3 \%)$.

- $70,7 \%$ tiveram algum tipo de experiência de trabalho na enfermagem durante a graduação, concomitante ao curso, em praticamente todos os anos estudados.

- o estágio remunerado foi a atividade extracurricular mais freqüentemente mencionada, totalizando $67,7 \%$ das respostas.

- $69,2 \%$ dos enfermeiros fizeram outros cursos após a graduação, sendo os de Especialização os mais mencionados $(41,0 \%)$.

- a área hospitalar foi a que mais absorveu os enfermeiros formados nesta Escola, no período considerado ( $76,2 \%$ das indicações).

- quanto à inserção em UTIs, um percentual considerável de enfermeiros destinou-se ao trabalho nestas Unidades; 47,0\% informaram que trabalham ou trabalharam em UTI, sendo que para $54,5 \%$ deles, esta foi a primeira área de atuação.

- dos enfermeiros que trabalham ou trabalharam em UTI, $52,8 \%$ não tinham experiência de atuação anterior nessa área.
- $64,1 \%$ receberam treinamento especifico para o início das atividades como enfermeiro de UTI.

- apesar do treinamento recebido, houve dificuldades para assumir as atividades, sendo as principais: a falta de destreza manual e de conhecimento teórico $(45,4 \%)$, o manuseio de equipamentos $(25,8 \%)$ e o relacionamento com as equipes de enfermagem e multiprofissional $(8,2 \%)$.

\section{REFERÊNCIAS BIBLIOGRÁFICAS}

BRASIL, Leis etc. Lei $\mathrm{n}^{\circ} 7498$ de 25 de Junho de 1986. Dispõe sobre a regulamentação do exercício da enfermagem e dá outras providências. Diário Oficial da União, Brasília, 26 Jun. de 1986. Seção 1, p. 9273-5.

BURNS, S.; HUTCHENS, A. L. New graduates in critical care: how long do they stay ?. J._Care Nurse, v. 12, n. 8, p.74-9, 1992.

CANFIELD, A. Controversy over clinical competences. Heart \& Lung, v. 19, p.197-9, 1982.

CHABOYER, W. et al. Critical care nurse's perceptions of their educational needs. Austr. J. Adv. Nurs, v.14, n.3, p.15-20, 1997.

GOMES, A.M. et al. Tendências no treinamento e reciclagem do pessoal de UTI. /Trabalho apresentado no III Encontro de Enfermagem em Terapia Intensiva, São Paulo, 1987.I

GOTTSCHALL, M. et al. Critical care orientation programs. Nurs.Manag., v.14, n.10,p.32-4, 1983.

IDE, C.A.C.; CHAVES, E.C. A distribuição geopolítica das unidades de terapia intensiva. Rev. Esc. Enf. USP, v.23, n.3 p.193-204, 1989.

IDE, C.A.C. et al. O seguimento do graduado em enfermagem. Rev.Esc.Enf.USP, v. 19, n.3, p. 195-211, 1985.

KIMURA, M.; MTYADAHIRA, A..M..K. Aspectos da assistência hospitalar no Município de São Paulo - a situação da assistência intensiva. Rev. Esc. Enf. USP, v.25, n.1, p.61-72, 1991.

KIMURA, M. et al. Avaliação sistematizada do processo ensinoaprendizagem em enfermagem médico-cirúrgica. Rev.Esc.Enf.USP, v.20, n.1, p. 27-38, 1986.

KOIZUMI, M.S. et al. Educação continuada da equipe de enfermagem nas UTIs do Município de São Paulo. Rev.Lat.Am.Enf., v. 6, n. 5, p. 33-41, 1998.

KREUTZ, I. A realidade hospitalar como primeira experiência profissional do enfermeiro. In: CONGRESSO BRASILEIRO DE ENFERMAGEM, 48, São Paulo, 1986. Resumos, Salvador, ABEn, 1986. p. 268.

KURCGANT, P. Formação e competência do enfermeiro de terapia intensiva. Enfoque, v.19, n. 1, p.4-6, 1991.

NAKAMAE, D. D. O perfil do estudante de enfermagem. São Paulo, 1976. 68p. Dissertação (Mestrado) - Escola de Enfermagem, Universidade de São Paulo.

OERMANN, M.H. Effectiveness of a critical care nursing course: preparing students for practice in critical care. Heart \& Lung, v.20, p.278-83, 1991.

SULLIVAN, S.; BREU, C. Survey of critical care nursing practice Part IV-staffing and training of intensive care unit personnel Heart \& Lung, v.11, n.3, p.237-41, 1982.

WIRCHOWISKI, H. C.; KUBSCH. S. How nurses react to and cope with the uncertainty of unfamiliar technology: validation for continuing education. J.Contin.Educ.Nurs., v.26, n.4, p.174-8, 1995. 


\section{ANEXO \\ QUESTIONÁRIO}

no.

\section{DADOS GERAIS}

-Idade: -Sexo:

Experiência de trabalho na enfermagem durante a graduação:

( ) não ( ) sim. Especifique.

-Cursos realizados após a formatura:

( ) nenhum

( ) doutorado

( ) mestrado

( ) atualização

( ) especialização. Especifique:

( ) aprimoramento. Especifique:...

( ) outros. Especifique:

\section{DADOS ESPECÍFICOS}

-Quanto às atividades desenvolvidas por você na área de enfermagem, após a formatura, assinale a alternativa que se aplica ao seu caso. Justifique se a sua resposta tiver um asterisco $\left(^{*}\right)$.

(Obs.: o termo trabalhar implica em ter vínculo empregatício)

( ) sempre trabalhei como enfermeiro(a)

() trabalhei como enfermeiro(a) e depois abandonei as atividades de enfermagem*

( ) trabalhei como enfermeiro(a), abandonei e depois retornei às atividades de enfermagem*

( ) apenas estudei e/ou fui bolsista na área de enfermagem*

( ) nunca trabalhei como enfermeiro(a) após a formatura*

( ) outros. Especifique:.

Justifique a alternativa assinalada:

Locais onde atuou após a formatura:

( ) Hospital

( ) Unidade Básica de Saúde

( ) Indústria/Empresa

( ) Escola. Especifique o nível

( ) outros. Especifique: 
Responda as questões a seguir, apenas se você atua ou atuou como enfermeiro(a) em UTI, após a formatura:

A U'TI foi a sua primeira área de atuação como enfermeiro?

( ) $\operatorname{sim}$

( ) não.

Tinha experiência anterior de trabalho em UTI?

( ) não

( ) sim . Como: ( ) auxiliar de enfermagem

( ) técnico de enfermagem

( ) estagiário remunerado

( ) outro. Especifique:

Recebeu treinamento específico para atuar como enfermeiro(a) na UTI?

( ) não

( ) sim. Especifique o tempo

Em linhas gerais, no que consistiu esse treinamento?

Quais foram as principais dificuldades que você enfrentou para assumir as funções como enfermeiro(a) de UTI? 